\title{
Spin Flips: Variation in the Orientation of Dark Matter Halos over their Merger Histories ${ }^{1}$
}

\author{
Philip E. Bett \\ Argelander-Institut für Astronomie, Universität Bonn, Auf dem Hügel 71, D-53121 Bonn, Germany
}

\begin{abstract}
Semi-analytic models of galaxy formation typically form the spheroidal components of galaxies ("bulges"), solely through galactic major mergers. However, it is possible that non-merger events (e.g. a "fly-by" by a smaller halo) can perturb a galaxy-halo system sufficiently to form a bulge. We present a preliminary investigation into the frequency of major changes in halo and galaxy spin direction, which could be signatures of such events.
\end{abstract}

Keywords: Galaxy formation, angular momentum, dark matter haloes, numerical simulations PACS: 98.62.Ai, 98.65.Fz, 95.35.+d

\section{FLIP AND MERGER EVENT DISTRIBUTION}

Using a large cosmological dark matter simulation, ${ }^{2}$ we look at the evolution of the haloes identified at $z=0$ by tracking their properties through their most-massive progenitors at each simulation output time $t_{i}$. We call the change in the properties of a halo between timesteps (e.g. from $t_{i-1} \rightarrow t_{i}$ ) an event, and we consider the distribution of events for all the selected ${ }^{3}$ haloes in the 46 timesteps from $z<6.2$. In particular, we compute the fractional halo mass change $\Delta \mu\left(t_{i}\right)=1-M\left(t_{i-1}\right) / M\left(t_{i}\right)$, and the angular momentum direction change $\cos \theta\left(t_{i}\right)=\left(\mathbf{j}\left(t_{i}\right) \cdot \mathbf{j}\left(t_{i-1}\right)\right) /\left(\left|\mathbf{j}\left(t_{i}\right)\right|\left|\mathbf{j}\left(t_{i-1}\right)\right|\right)$, in terms of the halo mass $M\left(t_{i}\right)$ and specific angular momentum vector $\mathbf{j}\left(t_{i}\right)$. We show the distribution of events in terms of $\Delta \mu$ and $\cos \theta$ in the left panel of Fig. 1, and their cumulative distributions in the middle and right panels. These show that major mergers are much more likely to than minor mergers to coincide with spin direction changes of more than a given angle, $\theta_{0}$. However, most events with significant direction change are minor mergers, not major.

\section{SPIN ORIENTATION IN A SINGLE OBJECT}

We use a high-resolution simulation of galaxy formation in a single halo (see [2] for details), to study the evolving relationship between halo and galaxy spin in more detail, over many hundreds of output times. In Fig. 2, we plot the evolution of the angular

\footnotetext{
${ }^{1}$ The original poster is available at http://www. astro. uni-bonn. de/ pbett/pub.html.

2 This is the "hMS" simulation of a $100 h^{-1} \mathrm{Mpc}$ box; see e.g. [1] for details.

${ }^{3}$ Each halo must have at least 1000 particles (i.e. $M \gtrsim 9.5 \times 10^{10} h^{-1} \mathrm{M}_{\odot}$ ), and have sufficiently large $\mathbf{j}$ that its orientation is not subject to discreteness bias: $\log _{10} \tilde{j} \equiv \log _{10}\left(|\mathbf{j}| / \sqrt{G M R_{\text {vir }}}\right) \geq-1.5$ (see [1]).
} 

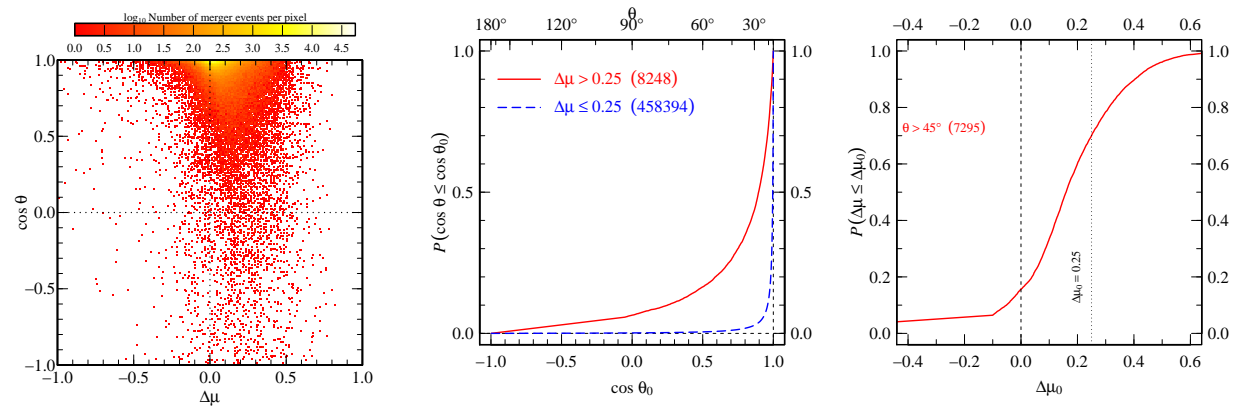

FIGURE 1. Left: Distribution of events in terms of fractional mass change and orientation change. Middle: Cumulative fraction of events with spin orientation change of at least $\theta_{0}$, for major mergers $(\Delta \mu>0.25)$ and minor mergers or accretion $(\Delta \mu \leq 0.25)$. Right: Cumulative fraction of events with $\Delta \mu \leq \Delta \mu_{0}$, for events where the spin direction changes by more than $\theta_{0}=45^{\circ}$.
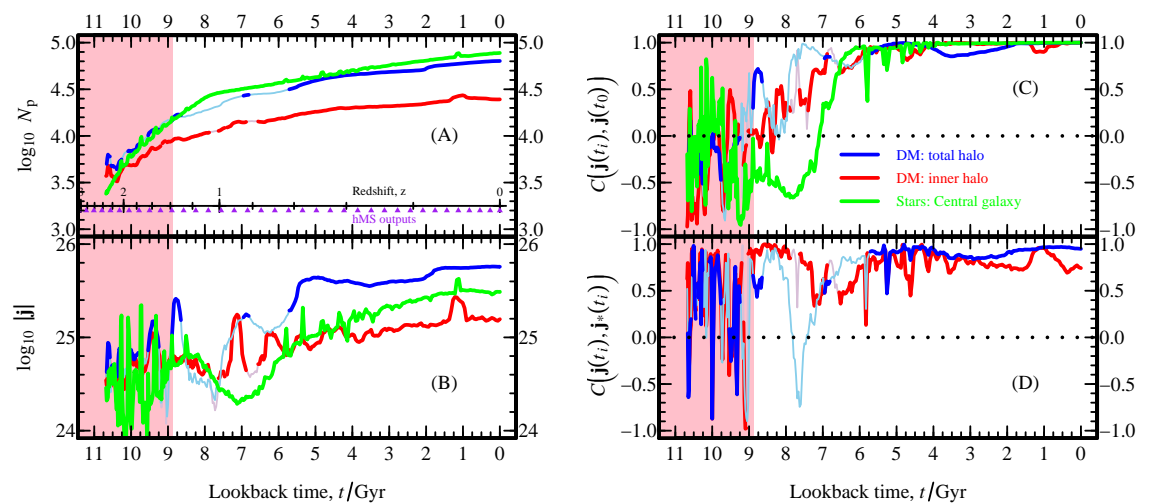

FIGURE 2. Evolution of a galaxy-halo system. In the pink shaded areas $(z \gtrsim 1.4)$, the system is not yet virialised. The four panels show (A) the number of particles, (B) the specific angular momentum magnitude $\left(\mathrm{m}^{2} / \mathrm{s}\right)$, and the angular momentum orientation with respect to both (C) the final time and (D) the galaxy at each timestep, where the $\operatorname{cosine} C(\mathbf{p}, \mathbf{q})=(\mathbf{p} \cdot \mathbf{q}) /(|\mathbf{p} \| \mathbf{q}|)$. The thin lines indicate when a component fails to satisfy our $\tilde{j}$ criterion, meaning its orientation is poorly defined.

momentum magnitudes and directions of the total halo dark matter, inner halo dark matter $\left(r \leq 0.25 R_{\mathrm{vir}}\right)$, and the stellar component of the central disc galaxy. Without a merger occurring, the halo flips $\sim 90^{\circ}$ at $t \sim 8 \mathrm{Gyr}$ (although its orientation is poorly defined at this point). The galaxy also flips by about $130^{\circ}$, but over a longer timescale. Afterwards, the galaxy and halo are well aligned. In this case, the disc survives, and the "flip" events are too slow to be seen by the analysis used in the previous section for the hMS simulation. In future work, we shall relate the timescales for spin flips to the masses and other properties of haloes and their progenitors, and characterise the frequency of these disturbing non-merger events.

\section{REFERENCES}

1. P. Bett, V. Eke, C. S. Frenk, A. Jenkins, and T. Okamoto, MNRAS submitted, arXiv:0906.2785 (2009)

2. T. Okamoto, V. R. Eke, C. S. Frenk, and A. Jenkins, MNRAS 363, 1299-1314 (2005). 